\title{
Concentraciones de 25 hidroxi vitamina D séricas y en lavado bronco-alveolar en pacientes con tuberculosis pulmonar
}

\author{
Levels of 25 hydroxy vitamin D of serum and broncho-alveolar lavage in patients \\ with pulmonary tuberculosis
}

\begin{abstract}
Cristhian Felipe Ramírez-Ramos ${ }^{1,3}$, Jhon Fredy Salamanca-Montilla',3, Emilio Herrera-Céspedes ${ }^{3}$, Juan Diego Rivera-Marín ${ }^{3}$, Paula Ximena Losada-Vanegas4, Juan David Areiza-Paramo3, David Ricardo Gutiérrez-Ramírez³, Nicolás Becerra-Meneces³, Jorge Luis Méndez Díaz ${ }^{3}$, María Isabel Cuellar Azuero, Alejandro Pinzón-Tovar ${ }^{5}$, Guido Lastra-Lastra ${ }^{3}$ y Giovani Lastra-González ${ }^{6}$
\end{abstract}

\author{
'Departamento de Cardiología Universidad, Surcolombiana, Neiva Colombia. \\ 2Departamento de Cardiología, Universidad de Antioquia, Medellín Colombia. \\ ${ }^{3}$ Universidad Nacional de Colombia, Bogotá D.C. \\ ${ }^{4}$ Departamento de Ciencias Básicas Biomédicas, Universidad de Antioquia, Medellín, Colombia. \\ ${ }^{5}$ Departamento de Medicina Interna y Endocrinología, Universidad Surcolombiana, Neiva, Colombia. \\ ${ }^{6}$ Departamento de Medicina Interna y Neumología, Universidad Surcolombiana, Neiva, Colombia.
}

Los autores expresan no tener conflictos de intereses con el presente estudio.

La financiación del examen de 25-hidroxi vitamina D la realizó el laboratorio BIOMERIEUX COLOMBIA con el aporte de dos kits de Vidas ${ }^{\circ} 25$ OH Vitamina D.

Recibido: 19 de enero de 2020 (segunda versión: 26 de noviembre de 2020) / Aceptado: 30 de diciembre de 2020

\section{Resumen}

Introducción: La alteración de la vitamina $\mathrm{D}$ es un factor de riesgo para enfermar de tuberculosis (TBC). Objetivo: Evaluar la concentración pulmonar y sérica del compuesto 25-hidroxi-vitamina D (25OHD) en pacientes con y sin TBC pulmonar. Metodología: Estudio de dos etapas: la primera parte fue de corte transversal, retrospectiva, y la segunda prospectiva. Se incluyeron $>18$ años a los que se les realizó fibrobroncoscopia por sospecha de TBC pulmonar y en quienes se confirmó la infección. Se tomaron como controles a pacientes con otro tipo de infección no TBC, y enfermedades no infecciosas para la primera etapa y controles infecciosos $\sin \mathrm{TBC}$ en la fase prospectiva. La medición de 25OHD se realizó mediante ELFA (ensayo de fluorescencia ligado a enzima). Se empleó la prueba de Kruskal-Wallis para evaluar asociación considerando significativo un valor de $\mathrm{p}<0,05$. Los datos se procesaron con el programa SPSS versión 23. Resultados: La muestra total fue de 77 pacientes (35 en la primera etapa y 42 en la segunda). Las características entre los grupos fueron homogéneas. Las concentraciones en suero (segunda fase) como en el lavado bronco-alveolar (primera y segunda fase) de 25OHD fueron más bajas en pacientes con TBC comparado con los controles e independientes de la concentración de calcio sérico (suero: $22,4 \mathrm{ng} / \mathrm{mL}$ vs $33 \mathrm{ng} / \mathrm{mL}, \mathrm{p}=0,006$ y lavado bronco-alveolar: $9,7 \mathrm{ng} / \mathrm{mL}$ vs $12,2 \mathrm{ng} / \mathrm{mL} ; \mathrm{p}=0,012$ ). Conclusiones: Hubo una diferencia significativa entre las concentraciones de 25OHD, tanto en suero como en lavado bronco-alveolar, en pacientes con TBC pulmonar con relación a sus controles.

Palabras clave: tuberculosis; vitamina D; lavado bronco-alveolar.

\section{Abstract}

Background: Alteration of vitamin D is a risk factor for tuberculosis (TB). Aim: To evaluate the pulmonary and serum levels of 25hydroxy vitamin $\mathrm{D}(25 \mathrm{OHD})$ in patients with and without pulmonary TB. Methods: Two-stage study: the first part was retrospective crosssectional and the second prospective. Those $>18$ years of age who underwent fiberoptic bronchoscopy for suspected pulmonary TB and in whom the infection was confirmed were included. Patients with another type of infection without TB and non-infectious diseases were taken as controls for the first stage and infectious controls without TB in the prospective phase. The measurement of 25OHD was performed by ELFA (enzyme-linked fluorescence assay). The Kruskal-Wallis test was used to evaluate association, considering a value of $p<0.05$ to be significant. The data were processed with the SPSS version 23 program. Results: The total sample was 77 patients (35 in the first stage and 42 in the second). The characteristics between the groups were homogeneous. Serum (second phase) and broncho-alveolar lavage (first and second phase) levels of 25OHD were lower in TB patients compared to controls and were independent of serum calcium level (serum: $22.4 \mathrm{ng} / \mathrm{mL}$ vs $33 \mathrm{ng} / \mathrm{mL}, \mathrm{p}=0.006$ and broncho-alveolar lavage: $9.7 \mathrm{ng} / \mathrm{mL}$ vs $12.2 \mathrm{ng} / \mathrm{mL} ; \mathrm{p}=0.012$ ). Conclusions: There was a significant difference between the levels of 25OHD in both serum and broncho-alveolar lavage in patients with pulmonary TB in relation to their controls.

Keywords: tuberculosis; vitamin D; broncho-alveolar lavage.

\section{Correspondencia a:}

Cristhian Felipe Ramirez-Ramos, https://orcid.org/0000-0002-3617-4585

cristhianramos1989@hotmail.com

cfrr89@gmail.com 


\section{Introducción}

L a tuberculosis (TBC) es una enfermedad infecciosa transmisible de gran importancia para la salud pública mundial. Durante los últimos dos siglos, ha causado el fallecimiento de aproximadamente un billón de personas, permaneciendo dentro de las primeras 10 causas de muerte en el mundo y como una importante etiología de discapacidad pulmonar crónica ${ }^{1}$. Por lo anterior, a lo largo de la historia se han diseñado múltiples estrategias para controlar esta enfermedad, aún no del todo exitosas, pese a los cambios recientes de la epidemiología mundial (tendencia a la disminución de la prevalencia) ${ }^{1}$.

En los últimos 30 años, los investigadores han descubierto el importante papel de la 25 -hidroxi vitamina $\mathrm{D}$ (25OHD), tanto en el sistema inmune innato, como en el adaptativo ${ }^{2}$. La $25 \mathrm{OHD}$ promueve la eliminación de Mycobacterium tuberculosis por medio de la destrucción por los macrófagos ${ }^{3}$. Por otro lado, la inmunidad innata es mediada a través del receptor nuclear de la 25OHD que estimula la síntesis de dos clases de péptidos antibacterianos: las catelicidinas y las $\beta$-defensinas producidas por los monocitos y las células epiteliales bronquiales. Dentro de los mecanismos puntuales, la transcripción de la catelecidina (codificada por el gep CAP-18 que es completamente dependiente de niveles suficientes de 25OHD), genera destrucción de las membranas micobacterianas en el fagosoma-lisosoma en los macrófagos ${ }^{4}$. La 25OHD previene la interrupción de la maduración del macrófago inducido por la micobacteria fagocitada, por medio de la activación de vía intracelular de la quinasa del 3 fosfatidil inositol. También, la activación del receptor tipo Toll-like 2 en los macrófagos y queratinocitos, convierte la 25OHD a su forma activa el 1,25 (OH)-hidroxi vitamina D. La 25OHD estimula la diferenciación de macrófagos y la producción de peróxido de hidrógeno y óxido nítrico, moléculas involucradas en la destrucción intracelular de M. tuberculosis. Algunos autores han sugerido que la actividad antimicobacteriana de la $25 \mathrm{OHD}$ puede operar a nivel inicial en el establecimiento de la infección como en la ingestión del bacilo por los macrófagos alveolares y las células dendríticas 5 . Muchos estudios han mostrado una asociación entre infección tuberculosa y polimorfismos de la $25 \mathrm{OHD}$, observación que ha reforzado la importancia de la molécula en la respuesta inmune a esta infección ${ }^{6,7}$.

Los ensayos clínicos han comprobado que la gran mayoría de pacientes con TBC se encuentran en estados de insuficiencia o deficiencia, asociando la concentración de vitamina $\mathrm{D}$ con el riesgo de sufrir la enfermedad ${ }^{8,9}$. En un estudio de casos y controles realizado en el año 2013, se compararon las concentraciones plasmáticas de 25OHD en pacientes que recibieron tratamiento para TBC respecto a pacientes que no habían presentado la enfermedad. Se encontró que los pacientes que recibieron tratamiento presentaban concentraciones más bajas de $25 \mathrm{OHD}(24,7$ $\mathrm{ng} / \mathrm{ml}$ vs $33,6 \mathrm{ng} / \mathrm{ml})^{10}$. En Corea se realizó un estudio similar de casos y controles en el que se compararon las concentraciones de 25OHD entre pacientes con TBC y personas sanas. Se concluyó que los pacientes con TBC tienen una mayor prevalencia de deficiencia de $25 \mathrm{OHD}$ (casos 9,86 ng/ml, IQR 7,19-14,15; controles 16,03 ng/ml, IQR 12,38-20,30, P <0,001); además, las concentraciones se incrementaron después del tratamiento ${ }^{11}$.

Resultados similares se encontraron en un metaanálisis publicado en el año 2015; se revisó un total de 15 estudios que incluían 1.440 casos y 2.558 controles. Los resultados indicaban que valores de $25 \mathrm{OHD}$ menores a $12,5 \mathrm{nmol} / \mathrm{L}$ incrementaban de forma significativa el riesgo de $\mathrm{TBC}(\mathrm{OR}=4,556,95 \% \mathrm{IC}=2,200-9,435$; $13-25 \mathrm{nmol} / \mathrm{L}: \mathrm{OR}=3.797,95 \% \mathrm{IC}=1,935-7,405)$. No se encontró asociación con concentraciones mayores de $51 \mathrm{nmol} / \mathrm{L}^{9}$.

En cuanto a las pruebas clínicas que evalúan las concentraciones de 25OHD en lavado bronco-alveolar (LBA), algunas iniciales constataron su producción por células extraídas mediante broncoscopia en pacientes con TBC, en enfermedades granulomatosas no relacionadas con TBC y en voluntarios sanos, encontrando diferencias ${ }^{(12,13)}$. Sin embargo, estos ensayos no midieron concentraciones del compuesto, ni comprobaron el estado sérico de los pacientes. Sólo hasta fecha reciente, una prueba realizada en La India determinó concentraciones séricas, en LBA, en pacientes con TBC, infección por VIH y personas sin dichas patologías, constatando diferencias significativas en los cuatro grupos comparados, siendo más bajos las concentraciones en los grupos que tenían $\mathrm{TBC}^{14}$.

A la fecha, no se cuentan con estudios que traten de correlacionar la producción local (pulmonar) de catelicidina como de 25OHD y su relación con el estado sérico de la misma en pacientes con TBC.

Se realizó el presente trabajo con el fin de evaluar las concentraciones de 250HD séricos y en el LBA, en pacientes con TBC pulmonar en un hospital de referencia del sur de Colombia.

\section{Materiales y Métodos}

El presente estudio de casos y controles, de intervención diagnóstica, se desarrolló en dos etapas.

\section{Primera fase}

Estudio de corte trasversal, de casos y controles. Se efectuó utilizando las muestras almacenas en el Laboratorio de Infección e Inmunidad de la Universidad Surcolombiana, obtenidas de un estudio previo titulado "Locally-secreted interleukin-6 is related with radiological severity in smear-negative pulmonary tuberculosis" 
sospecha de TBC pulmonar y en quienes se confirmó posteriormente la infección, que aceptaran participar y dieran su consentimiento informado. Se excluyeron pacientes con historia de neoplasia pulmonar primaria activa o tratada.

El protocolo de la broncoscopia y el LBA fue el descrito previamente. La cantidad de muestra de LBA fueron $10 \mathrm{~mL}$ y además se tomaron $10 \mathrm{~mL}$ de sangre para la medición sérica del compuesto y otros parámetros del metabolismo del calcio. Las muestras se trasportaron al laboratorio prontamente después de la toma para ser centrifugadas y procesadas de inmediato.

El seguimiento de los pacientes se hizo desde el procedimiento y durante la estancia hospitalaria cada $72 \mathrm{~h}$ hasta el alta.

Las muestras se procesaron inmediatamente por medio de la técnica según indicaciones del fabricante (ELFA] BIOMERIEUX COLOMBIA kit de Vidas $^{\circledR} 25$ OH Vitamina D).

Para el análisis de los resultados de la cohorte prospectiva se dividieron los pacientes en dos grupos: caso de $T B C$ paciente en quien se confirmara la infección por medio de cultivo o RPC y controles pacientes en quien se realizó la broncoscopia por otra indicación o enfermedad infecciosa con el requisito de haberse excluido infección por TBC utilizando los métodos mencionados.

Los pacientes en ambos grupos fueron adicionalmente analizados teniendo puntos de corte de $25 \mathrm{OHD}$ entre deficiencia (menor $20 \mathrm{ng} / \mathrm{ml}$ ), insuficiencia (< de $30 \mathrm{ng} /$ dl) y suficiencia (>30 ng/ml).

Mediante un cuestionario diseñado por los investigadores se tomaron datos socio-demográficos, antropométricos (peso, talla, índice de masa corporal), antecedentes patológicos (enfermedades crónicas, inmunosupresión farmacológica, historia de trasplante, historia de neoplasia, historia de infección por VIH, historia de contacto con pacientes con infección tuberculosa), síntomas de presentación inicial, parámetros de laboratorio (concentración sérica de calcio y albúmina) y además, el tipo de proceso infeccioso por el que se indicó la broncoscopia para el grupo de control.

Se generaron estadísticas descriptivas (frecuencias y proporciones) para las variables cualitativas, medidas de tendencia central (media, mediana), medida de dispersión (desviación estándar) y medidas de posición (mínimo, máximo y rango). Para identificar la asociación entre variables se empleó la prueba Kruskal Wallis. En todos los casos se tuvo en cuenta un nivel de significancia estadística cuando el valor $\mathrm{p}<0,05$. Para todos los análisis de datos se utilizó el paquete estadístico SPSS versión 23.

El presente estudio fue aprobado por el Comité de Ética del Hospital Universitario Hernando Moncaleano. De acuerdo con la resolución número 8430 de 1993 de Colombia, articulo 11, el presente estudio se clasifica 
como investigación con riesgo mínimo debido a que es un estudio ambispectivo que emplea un procedimiento común para registro de datos. Vale la pena mencionar que la broncoscopia se realizó a los pacientes por indicación de su estancia hospitalaria y no como objetivo primario de la obtención de muestras para el estudio.

\section{Resultados}

\section{Primera fase (corte transversal retrospectiva)}

Un total de 35 muestras de LBA se incluyeron en el análisis. Se realizaron tres grupos de comparación: pacientes con TBC pulmonar confirmada (n: 9), controles infecciosos (n: 12) y controles no infecciosos (n: 14).

La mayoría de los pacientes en los tres grupos eran de sexo masculino, con promedio de edades de 33, 59 y 57 años, respectivamente. Los datos se muestran en la Tabla 1. La hipertensión arterial esencial fue la co-morbilidad prevalente en el grupo de controles infecciosos sin TBC y en los controles no infecciosos. En los pacientes con TBC se encontró que un tercio tenía infección por VIH como co-morbilidad principal, con una prevalencia alta comparado a los grupos de control.

Se detectaron concentraciones basales por medio de la prueba utilizada de 25OHD en una mayor proporción en el grupo de controles no infecciosos (57\%) comparado con los pacientes con $\mathrm{TBC}$, donde sólo $18 \%$ de la población presentó concentraciones detectables de 25OHD por la prueba utilizada. Como se puede observar en la Tabla 2, en los pacientes con concentraciones basales detectables del compuesto, dichas concentraciones fueron más bajas en los pacientes con TBC comparando con los controles, tanto infecciosos sin TBC como los no infecciosos; sin embargo, sin alcanzar significancia estadística.

\section{Segunda fase (cohorte prospectiva)}

Para la fase prospectiva con los criterios de inclusión del estudio se reclutaron 42 pacientes. El análisis se realizó en dos grupos: casos confirmados de TBC (n: 18) y controles infecciosos (n: 24). En relación con las características basales de la población, no hubo diferencias entre los grupos, siendo estos homogéneos (Tabla 3); la mayoría fue de sexo masculino, con edades medias de 45 y 52 años, respectivamente. De los 18 casos de TBC, 17 fueron diagnosticados por cultivo y uno mediante RPC. De los 24 controles infecciosos la principal indicación del estudio fue neumonía adquirida en comunidad (Tabla 4).

En los pacientes con diagnóstico de TBC, las manifestaciones clínicas más frecuentes fueron: tos $(88,9 \%)$ y fiebre $(83,3 \%)$ seguidas de diaforesis $(56 \%)$, sudoración $(55,6 \%)$, pérdida de peso $(55,6 \%)$, disnea $(55 \%)$, hipo-

\begin{tabular}{|c|c|c|c|c|c|}
\hline & $\begin{array}{l}\text { Sexo } \\
\text { n (\%) }\end{array}$ & $\begin{array}{l}\text { Edad } \\
\text { (años)* }\end{array}$ & $\underset{\left(\mathrm{kg} / \mathrm{m}^{2}\right)^{*}}{\mathrm{IMC}}$ & $\begin{array}{c}\text { Co-infección por VIH } \\
(\%)\end{array}$ & Principal co-morbilidad (\%) \\
\hline $\begin{array}{l}\text { Tuberculosis, } \\
\mathrm{n}=9\end{array}$ & $\begin{array}{l}\text { Hombres: } \\
5(55,5 \%)\end{array}$ & 33 años & 20,4 & $33 \%$ & $\begin{array}{l}\text { EPOC }(111 \%) \\
\text { Enfermedad renal crónica }(11,1 \%)\end{array}$ \\
\hline $\begin{array}{l}\text { Controles infecciosos no TBC, } \\
\mathrm{n}=12\end{array}$ & $\begin{array}{l}\text { Hombres: } \\
7(58,3 \%)\end{array}$ & 59 años & 24,0 & $16 \%$ & $\begin{array}{l}\text { HTA }(41, .6 \%) \\
\text { EPOC (33\%) }\end{array}$ \\
\hline $\begin{array}{l}\text { Controles no infecciosos, } \\
n=14\end{array}$ & $\begin{array}{l}\text { Hombres: } \\
8(57,1 \%)\end{array}$ & 57 años & 22,8 & $0 \%$ & $\begin{array}{l}\operatorname{HTA}(22,2 \%) \\
\text { Enfermedad renal crónica }(22,2 \%) \\
\text { EPOC }(11,1 \%)\end{array}$ \\
\hline
\end{tabular}

*Promedio. IMC: índice de masa corporal. EPOC: enfermedad pulmonar obstructiva crónica. HTA: hipertensión arterial sistémica.

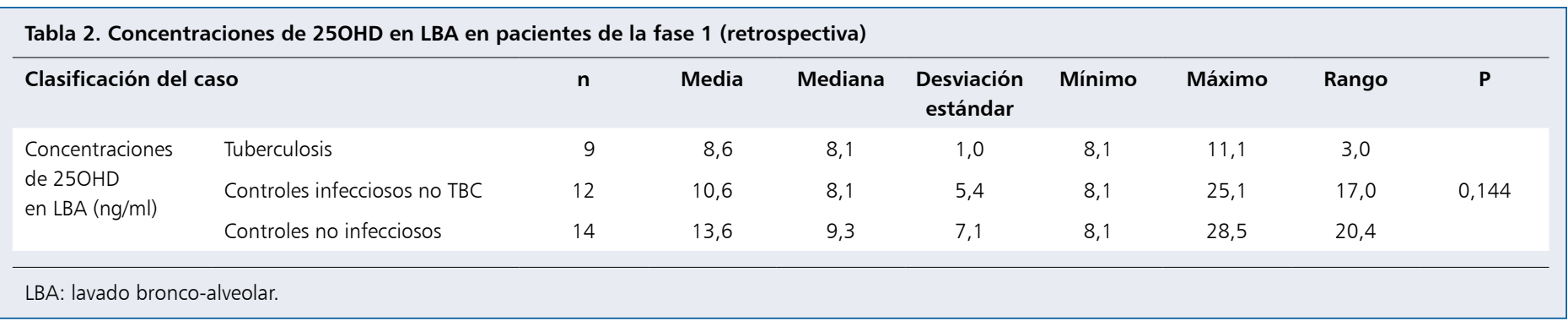




\section{Investigación Clínica}

rexia $(38 \%)$ y hemoptisis $(22 \%)$. En relación con los antecedentes relevantes, dos de estos pacientes tenían enfermedad renal crónica sin estar en hemodiálisis, un paciente linfoma no Hodgkin, un paciente historia de trasplante renal en tratamiento y uno historia de TBC. Ninguno tenía antecedente de infección por VIH y a uno de ellos se le diagnosticó esta afección en la estancia hospitalaria mediante prueba ELISA (Tabla 3).

Con respecto a los resultados primarios se encontraron concentraciones de $25 \mathrm{OHD}$ más bajos en suero en los pacientes con TBC en comparación a los controles infecciosos sin TBC (Tabla 5). La media del valor sérico en los casos de TBC fue de $22,4 \mathrm{ng} / \mathrm{mL}$ (95\% IC 14,2$44,4 \mathrm{ng} / \mathrm{mL}$ ) y en los controles de $33 \mathrm{ng} / \mathrm{mL}$ (95\% IC $13,2-50,4 \mathrm{ng} / \mathrm{mL})$ con una diferencia significativamente estadística $(\mathrm{p}=0,006)$.

En el análisis teniendo en cuenta puntos de corte entre deficiencia (menor $20 \mathrm{ng} / \mathrm{ml}$ ), insuficiencia (< de $30 \mathrm{ng} /$ dl) y suficiencia ( $>30 \mathrm{ng} / \mathrm{ml}), 83,3 \%$ de los pacientes del grupo de TBC se encontraban en estados de insuficiencia $(22,2 \%)$ o deficiencia $(61,1 \%)$ comparado con $29,2 \%$ de los pacientes de su grupo de control [insuficiencia (12,5\%) y deficiencia $(15,7 \%)]$.

La media de las concentraciones de $25 \mathrm{OHD}$ en el LBA de los pacientes con TBC fue de $9,7 \mathrm{ng} / \mathrm{mL}(95 \% \mathrm{IC}$ $8,1-22,4 \mathrm{ng} / \mathrm{mL})$ y en los controles de $12,2 \mathrm{ng} / \mathrm{mL}(95 \%$ IC $8,1-21,7 \mathrm{ng} / \mathrm{mL}$ ) con una diferencia estadísticamente significativa entre los grupos $(p=0,012)$.

No hubo diferencia significativa entre el perfil de metabolismo de calcio en los diferentes grupos evaluados. La media en los niveles de calcio sérico fue de $8,6 \mathrm{mg} / \mathrm{dL}$ (grupo TBC), y 8,3 mg/dL (grupo control) $(\mathrm{p}=0,191)$.

La media de los niveles de albúmina fue de 2,9 g/ dL y 2,7 g/dL para los casos de TBC y el grupo control, respectivamente $(\mathrm{p}=0,428)$ (Tabla 5).

La mayoría de los pacientes estaban vivos al final de la hospitalización (80,8\%), y los demás pacientes $(19,2 \%)$ fallecieron casi todos en unidad de cuidados intensivos. De las muertes que se presentaron, cinco fueron en el grupo de controles infecciosos sin TBC y tres en el grupo de pacientes con TBC confirmada.

\section{Discusión}

Presentamos los resultados clínicos, parámetros de laboratorio y concentraciones de 25OHD medidas en LBA y sanguíneas de pacientes con TBC pulmonar confirmada.

El presente estudio demostró, tanto en el primer análisis como en la cohorte prospectiva, que de manera local se produce $25 \mathrm{OHD}$ y refuerza la participación de la molécula de $25 \mathrm{OHD}$ en la fisiopatología de la TBC. Interesantemente, esto no parece depender de las demás sustancias que influyen en el metabolismo mineral (calcio).

\begin{tabular}{|c|c|c|}
\hline & $\begin{array}{l}\text { Controles } \\
\text { infecciosos } \\
\text { n (\%) }\end{array}$ & $\begin{array}{c}\text { Tuberculosis } \\
\text { n (\%) }\end{array}$ \\
\hline Hombres & $14(58,3)$ & $13(72,2)$ \\
\hline Edad (años) & 52 & 45 \\
\hline Peso (kg) & 61,3 & 62,4 \\
\hline IMC $\left(\mathrm{kg} / \mathrm{m}^{2}\right)$ & 23,4 & 22,2 \\
\hline Antecedente de contacto con personas con TBC & $23(95,8)$ & $16(88,9)$ \\
\hline Hipertension arterial esencial & $5(20,8)$ & $4(22,2)$ \\
\hline Diabetes mellitus tipo 2 & $4(16,7)$ & $3(16,7)$ \\
\hline EPOC & $7(29,2)$ & $4(22,2)$ \\
\hline Enfermedad renal crónica (TFGc $<60 \mathrm{mil} / \mathrm{min} / 1,73$ m² s.c.) & $5(20,8)$ & $2(11,1)$ \\
\hline Terapia de reemplazo renal & $1(4,2)$ & 0 \\
\hline Enfermedad autoinmune & $2(8,3)$ & 0 \\
\hline Antecedente de TBC & $1(4,2)$ & $1(5,6)$ \\
\hline Tratamiento para TBC previo & $1(4,2)$ & $1(5,6)$ \\
\hline Infección por VIH & $1(4,2)$ & 0 \\
\hline Neoplasia hemato-linfoide & $5(20,8)$ & $1(5,6)$ \\
\hline Leucemia mieloide aguda & $1(4,2)$ & 0 \\
\hline Leucemia mieloide crónica & $1(4,2)$ & 0 \\
\hline Linfoma no Hodgkin & $2(8,3)$ & $1(5,6)$ \\
\hline Mieloma múltiple & $1(4,2)$ & 0 \\
\hline Historia de trasplante & $2(8,3)$ & $1(5,6)$ \\
\hline Trasplante renal & $2(8,3)$ & $1(5,6)$ \\
\hline Fallecido al alta hospitalaria & $5(20,8)$ & $3(16,7)$ \\
\hline Vivo al alta hospitalaria & $19(79,2)$ & $15(83,3)$ \\
\hline Prueba de VIH en hospitalización índice & $15(62,5)$ & $16(88,9)$ \\
\hline Prueba para VIH positiva & $1 \quad(4,2)$ & $1 \quad(5,6)$ \\
\hline
\end{tabular}

\begin{tabular}{lc|}
\hline Tabla 4. Diagnósticos clínicos en el grupo control con infecciones no TBC \\
\hline & Controles infecciosos no TBC \\
\hline Bronquitis infecciosa & $2(8,3)$ \\
EPOC exacerbado & $3(12,5)$ \\
Neumonía adquirida en comunidad & $13(54,2)$ \\
Neumonía asociada a ventilador & $1(4,2)$ \\
Neumonía asociada al cuidado de la salud & $3(12,6)$ \\
\hline Traqueítis & $2(8,3)$ \\
\hline
\end{tabular}




\begin{tabular}{|c|c|c|c|c|c|c|}
\hline Variable & & $\mathbf{n}$ & Media & Mediana & Desviación estándar & p \\
\hline \multirow[t]{2}{*}{ Concentraciones de $25 \mathrm{OHD}$ en LBA ( $\mathrm{ng} / \mathrm{ml})$} & Controles infecciosos no TBC & 24 & 12,2 & 11,5 & $4,6(8,1-21,7)$ & \multirow{2}{*}{0,012} \\
\hline & Tuberculosis & 18 & 9,7 & 8,1 & $3,6(8,1-22,4)$ & \\
\hline \multirow[t]{2}{*}{ Concentraciones de $250 \mathrm{HD}$ en suero $(\mathrm{ng} / \mathrm{ml})$} & Controles infecciosos no TBC & 24 & 33,0 & 35,2 & $10,3(13,2-50,4)$ & \multirow{2}{*}{0,006} \\
\hline & Tuberculosis & 18 & 22,4 & 19,5 & $8,4(14,2-44,4)$ & \\
\hline \multirow[t]{2}{*}{ Concentraciones de calcio sérico (mg/dL) } & Controles infecciosos no TBC & 24 & 8,3 & 8,1 & $0,6(7,1-9,3)$ & \multirow{2}{*}{0,191} \\
\hline & Tuberculosis & 18 & 8,6 & 8,7 & $0,7(7,1-10,2)$ & \\
\hline \multirow[t]{2}{*}{ Concentraciones de calcio iónico (mmol/L) } & Controles infecciosos no TBC & 24 & 1,0 & 1,0 & $0,1(0,8-1,2)$ & \multirow{2}{*}{0,534} \\
\hline & Tuberculosis & 18 & 1,0 & 1,0 & $0,1(0,9-1,2)$ & \\
\hline \multirow[t]{2}{*}{ Concentración de albumina (mg/dL) } & Controles infecciosos no TBC & 24 & 2,7 & 2,5 & $0,9(1,3-4,6)$ & \multirow{2}{*}{0,428} \\
\hline & Tuberculosis & 18 & 2,9 & 2,9 & $0,8(1,1-4,0)$ & \\
\hline \multirow[t]{2}{*}{ Calcio corregido a albumina (mg/dL) } & Controles infecciosos no TBC & 24 & 9,3 & 9,4 & $0,8(7,0-10,6)$ & \multirow{2}{*}{0,266} \\
\hline & Tuberculosis & 18 & 9,6 & 9,6 & $0,8(8,2-10,8)$ & \\
\hline
\end{tabular}

Se encontró que son frecuentes los estados de alteración de la $25 \mathrm{OHD}$ (deficiencia o insuficiencia) en los pacientes con TBC.

De las características de la población, vale la pena mencionar que, si bien no se encontró alta prevalencia de condiciones o factores de riesgo de TBC (infección por $\mathrm{VIH}$, diabetes mellitus, trasplante, enfermedad renal crónica), dichas características fueron similares en los dos grupos finales de comparación teniendo poblaciones homogéneas.

Múltiples pruebas han demostrado que los pacientes con TBC pulmonar tienen concentraciones séricas más bajas de 25OHD comparativamente con controles sanos $\mathrm{y}$, además, que se comporta como un factor de riesgo o de susceptibilidad para dicha infección, algo que concuerda con lo que se encontró en la población de pacientes con TBC, no siendo así para el grupo de control.

A la fecha, pese a que se ha comprobado la producción pulmonar de $25 \mathrm{OHD}$, no se ha estandarizado la técnica ni la concentración basal del compuesto. Los primeros estudios realizados al respecto fueron planteados por Cadranel y cols. ${ }^{12}$, quienes establecieron que las células pulmonares eran capaces de producir el metabolito activo de la $25 \mathrm{OHD}$, tanto en muestras obtenidas por medio de LBA de pacientes con TBC como de sus controles, pudiendo documentar por primera vez la producción extra-renal de dicha sustancia. Sin embargo, no se estableció si estas concentraciones estaban relacionadas con el estado sérico del paciente.

En la primera parte de esta investigación se demostró que los pacientes con TBC tenían concentraciones detectables del compuesto en LBA en un porcentaje menor de pacientes ( $18 \%$ de personas con TBC vs $57 \%$ controles no infecciosos sin TBC con concentraciones medidas por arriba del límite de detección). En las muestras de pacientes en quienes se detectaron concentraciones de $25 \mathrm{OHD}$ en el LBA, las concentraciones fueron inferiores en el grupo TBC al compararlo con sus controles infecciosos sin TBC y los no infecciosos $(8,55 \mathrm{ng} / \mathrm{mL}$ vs $10,55 \mathrm{ng} / \mathrm{mL}$ vs $13,57 \mathrm{ng} / \mathrm{mL}$ ) lo que coincide con los reportado en La India en un estudio con una metodología muy similar, pese a la diferencia en la técnica utilizada para la medición ${ }^{10}$.

En la fase prospectiva encontramos, con una diferencia estadísticamente significativa, que los pacientes con TBC pulmonar tenían concentraciones séricas inferiores comparadas con los controles infecciosos $(22,4 \mathrm{ng} / \mathrm{mL}$ vs $33,0 \mathrm{ng} / \mathrm{mL}$ ), datos que concuerdan con lo reportando en la literatura científica ${ }^{8,14}$. Esto fue independiente de la concentración de calcio sérico, pues en los grupos no se detectó en este paramento una diferencia significativa, factor que puede influir en la concentración de 250HD.

En cuanto a las concentraciones detectadas en el LBA, se apreció nuevamente que los pacientes con TBC pulmonar tenían unas concentraciones más bajas respecto a su grupo de comparación $(9,7 \mathrm{ng} / \mathrm{mL}$ vs $12,2 \mathrm{ng} / \mathrm{mL})$; estos datos coinciden nuevamente con lo descrito por Sinha y cols. ${ }^{14}$.

Encontramos una posible relación entre las concentraciones séricas y en el LBA de los grupos de estudio, algo 
Dentro de las limitaciones del presente trabajo hay que mencionar:

- El número de pacientes con TBC y controles es bajo; sin embargo, esto es propio a la naturaleza del diseño (pacientes que tenían indicación de fibrobroncoscopia).

- La prueba utilizada para las mediciones de vitamina D tiene un límite inferior de detección $(8,1 \mathrm{ng} / \mathrm{mL})$ que se pudiera superar con otras técnicas empleadas en los ensayos mencionados (ELISA) mejorando la capacidad de detección en otros sitios diferentes a muestras séricas

\section{Conclusiones}

Los resultados del presente estudio están en concordancia con lo descrito recientemente de que en el pulmón hay concentraciones detectables de 250HD que, además, se relacionan con el estado sérico del paciente; dicho de otra manera, el estado sérico influye directamente en las concentraciones periféricas de este compuesto.

Aún queda por esclarecer si esta asociación, influye en la producción de moléculas que tienen un papel importante en la patogénesis de la infección y que son totalmente vitamina D-dependientes (catelicidinas y defensinas).

Agradecimientos. Los autores expresan su agradecimiento a todo el personal del laboratorio clínico del hospital en dirección de la doctora Alicia Cortes, por su invaluable apoyo en la presente investigación. A Yulieth Patricia, Auxiliar del Departamento de Neumología quién colaboró en la recolección de muestras de los pacientes.

\section{Referencias bibliográficas}

1.- Dheda K, Barry C E, Maartens G. Tuberculosis. Lancet. 2016; 387 (10024): 1211-26. doi: 10.1016/S0140-6736(15)00151-8.

2.- Hewison M. Vitamin D and the immune system: new perspectives on an old theme. Rheum Dis Clin North Am. 2012; 38 (1): 125 39. doi: 10.1016/j.ecl.2010.02.010.

3.- Bruns H, Stenger S. New insights into the interaction of Mycobacterium tuberculosis and human macrophages. Future Microbiol. 2014; 9 (3): 327-41. doi: 10.2217/fmb.13.164.

4.- Aranow C. Vitamin D and the immune system. J Investig Med. 2011; 59 (6): 881-6. doi: 10.231/JIM.0b013e31821b8755.

5.- Liu P T, Stenger S, Li H, Wenzel L, Tan B H, Krutzik S R, et al. Toll-like receptor triggering of a vitamin D-mediated human antimicrobial response. Science. 2006; 311 (5768): 1770-3. doi: 10.1126/science.1123933.

6.- Areeshi M Y, Mandal R K, Akhter N, Panda
A K, Haque S. Evaluating the association between TaqI variant of vitamin D receptor gene and susceptibility to tuberculosis: a meta-analysis. Toxicol Int. 2014; 21 (2): 140-7. doi: 10.4103/0971-6580.139791.

7.- Salamon H, Bruiners N, Lakehal K, Shi L, Ravi J, Yamaguchi K D, et al. Cutting edge: vitamin $\mathrm{D}$ regulates lipid metabolism in Mycobacterium tuberculosis infection. J Immunol. 2014; 193 (1): 30-4. doi: 10.4049/jimmunol.1400736.

8.- Nnoaham K E, Clarke A. Low serum vitamin D levels and tuberculosis: a systematic review and meta-analysis. Int J Epidemiol. 2008; 37 (1): 113-9. doi: 10.1093/ije/dym247.

9.- Zeng J, Wu G, Yang W, Gu X, Liang W, Yao Y, et al. A serum vitamin $D$ level $<25 \mathrm{nmol} / 1$ pose high tuberculosis risk: a meta-analysis. PLoS One. 2015; 10 (5): e0126014. doi: 10.1371/ journal.pone. 0126014 .

10.- Huaman M A, Sterling T R, Shepherd B E, Fiske C T. 25-hydroxyvitamin D levels after recovery from tuberculosis: insights into pathogenesis. Tuberculosis (Edinb). 2014; 94 (1): 51-4. doi: 10.1016/j.tube.2013.10.009.

11.- Hong J Y, Kim S Y, Chung K S, Kim E Y, Jung J Y, Park M S, et al. Association between vitamin $\mathrm{D}$ deficiency and tuberculosis in a Korean population. Int J Tuberc Lung Dis. 2014; 18 (1): 73-8. doi: 10.5588/ijtld.13.0536.

12.- Cadranel J, Hance AJ, Milleron B, Paillard F, Akoun G M, Garabedian M. Vitamin D metabolism in tuberculosis. Production of $1,25(\mathrm{OH}) 2 \mathrm{D} 3$ by cells recovered by bronchoalveolar lavage and the role of this metabolite in calcium homeostasis. Am Rev Respir Dis. 1988; 138 (4): 984-9. doi: 10.1164/ ajrccm/138.4.984.

13.- Cadranel J, Garabedian M, Milleron B, Guillozo H, Akoun G, Hance A J. $1,25(\mathrm{OH}) 2 \mathrm{D} 2$ production by $\mathrm{T}$ lymphocytes and alveolar macrophages recovered by lavage from normocalcemic patients with tuberculosis. J Clin Invest. 1990; 85 (5): 158893. doi: 10.1172/JCI114609. 
14.- Sinha S, Gupta K, Mandal D, Das B K, Pandey R M. Serum and bronchoalveolar lavage fluid $25(\mathrm{OH})$ vitamin D3 levels in HIV-1 and tuberculosis: a cross-sectional study from a tertiary care center in North India. Curr HIV Res. 2018; 16 (2): 167-73. doi: 10.2174/1570162X16666180528112924.

15.- Losada P X, Perdomo-Celis F, Castro M, Salcedo C, Salcedo A, DeLaura I, et al. Locally-secreted interleukin-6 is related with radiological severity in smear-negative pulmonary tuberculosis. Cytokine. 2020; 127 : 154950. doi: 10.1016/j.cyto.2019.154950.

16.- Meyer K C, Raghu G, Baughman R P, Brown K K, Costabel U, du Bois R M, et al. An official American Thoracic Society clinical practice guideline: the clinical utility of bronchoalveolar lavage cellular analysis in interstitial lung disease. Am J Respir Crit Care Med. 2012; 185 (9): 1004-14. https://doi.org/10.1164/ rccm.201202-0320ST.

17.- Yamshchikov A V, Kurbatova E V, Kumari M, Blumberg H M, Ziegler T R, Ray S M, et al. Vitamin D status and antimicrobial peptide cathelicidin (LL-37) concentrations in patients with active pulmonary tuberculosis. Am J Clin Nutr. 2010; 92 (3): 603-11. doi: 10.3945/ ajen.2010.29411.

18.- Majewski K, Agier J, Kozłowska E, Brzezińska-Błaszczyk E. Status of cathelicidin IL-37, cytokine TNF, and vitamin $\mathrm{D}$ in patients with pulmonary tuberculosis. J Biol Regul Homeost Agents. 2018; 32 (2): 321-5. PMID: 29685013.

19.- Sinclair D, Abba K, Grobler L, Sudarsanam T D. Nutritional supplements for people being treated for active tuberculosis. Cochrane Database Syst Rev. 2011; (11): CD006086. doi: 10.1002/14651858.CD006086.pub3.

20.- Coussens A K, Wilkinson R J, Hanifa Y, Nikolayevskyy V, Elkington P T, Islam K, et al. Vitamin D accelerates resolution of inflammatory responses during tuberculosis treatment. Proc Natl Acad Sci U S A. 2012; 109 (38): 15449-54. doi: 10.1073/ pnas. 1200072109 .

21.- Salahuddin N, Ali F, Hasan Z, Rao N, Aqeel M, Mahmood F. Vitamin D accelerates clinical recovery from tuberculosis: results of the SUCCINCT Study [Supplementary cholecalciferol in recovery from tuberculosis]. A randomized, placebo-controlled, clinical trial of vitamin $\mathrm{D}$ supplementation in patients with pulmonary tuberculosis'. BMC Infect Dis. 2013; 13: 22. doi: 10.1186/1471-2334-13-22.

22.- Jolliffe D A, Ganmaa D, Wejse C, Raqib R, Haq M A, Salahuddin N, et al. Adjunctive vitamin $\mathrm{D}$ in tuberculosis treatment: meta-analysis of individual participant data. Eur Respir J. 2019; 53 (3) 1802003. doi: 10.1183/13993003.020032018.

23.- Ganmaa D, Munkhzul B, Fawzi W, Spiegelman D, Willett W C, Bayasgalan P, et al. High-dose vitamin D3 during tuberculosis treatment in Mongolia. A randomized controlled trial. Am J Respir Crit Care Med. 2017; 196 (5): 628-37. doi: 10.1164/ rccm.201705-0936OC.

24.- Wu H X, Xiong X F, Zhu M, Wei J, Zhuo K Q, Cheng D Y. Effects of vitamin D supplementation on the outcomes of patients with pulmonary tuberculosis: a systematic review and meta-analysis. BMC Pulm Med. 2018; 18 (1): 108. doi: 10.1186/s12890-0180677-6. 